\title{
Frontières
}

\section{Rire à la face de la mort}

\section{Denyse Therrien}

Volume 19, numéro 2, printemps 2007

\section{Penser sa mort?}

URI : https://id.erudit.org/iderudit/017498ar

DOI : https://doi.org/10.7202/017498ar

Aller au sommaire du numéro

Éditeur(s)

Université du Québec à Montréal

ISSN

1180-3479 (imprimé)

1916-0976 (numérique)

Découvrir la revue

Citer cet article

Therrien, D. (2007). Rire à la face de la mort. Frontières, 19(2), 53-58.

https://doi.org/10.7202/017498ar

Ce document est protégé par la loi sur le droit d'auteur. L'utilisation des services d'Érudit (y compris la reproduction) est assujettie à sa politique d'utilisation que vous pouvez consulter en ligne.

https://apropos.erudit.org/fr/usagers/politique-dutilisation/
Cet article est diffusé et préservé par Érudit.

Érudit est un consortium interuniversitaire sans but lucratif composé de l’Université de Montréal, l'Université Laval et l'Université du Québec à Montréal. Il a pour mission la promotion et la valorisation de la recherche. https://www.erudit.org/fr/ 


\section{RIRE \\ À LA FACE DE LA MORT}

\section{Denyse Therrien, Ph. D. chercheure et praticienne en arts visuels, UQAM.}

La mort n'est pas - loin s'en faut - un sujet privilégié par les humoristes. Que ce soit en littérature ou au cinéma, la mort appelle le drame, convoque les spectateurs à partager les émotions, le ressenti, le vécu des personnages mis en scène et, dans la plupart des cas, garantit le succès du film ou du livre, surtout si la mort survient suite à une maladie mortelle. Ainsi en va-t-il des nombreuses œuvres cinématographiques qui mettent en scène des personnages atteints d'un cancer ou, plus récemment, du sida ${ }^{2}$, et des livres - récits de l'agonie ou témoignages de survivants à de telles maladies.

Vaincre la maladie mortelle relève du miracle ou de l'exploit - selon le degré de sa foi - et semble valoir d'être raconté pour nourrir l'espoir non seulement des victimes de telles maladies, mais de l'ensemble des gens car, on le sait, cela n'arrive pas qu'aux autres. Il est donc inhabituel que la mort fasse l'objet du rire. C'est pourquoi les rares cas de figure en cette matière offrent un grand intérêt, le rire étant selon plusieurs, le meilleur remède à toute maladie, fût-elle déclarée incurable. Par contre, il nous semble important d'apporter une réserve, ou plutôt, de nuancer le rôle de l'humour chez les patients qui souffrent d'une maladie physique par rapport à ceux atteints de maladie mentale, et de différencier l'humour et la blague.
MARXEST MORT,

FREUD EST MORT, DIEU EST MORT. ET MOI-MÊME, CELA NE VA PAS TRĖS FORT...

WOODY ALLEN, CITÉ DANS WOODROW, 2000, P. $184^{1}$.

L'humour est d'abord et avant tout moquerie de soi, alors que, la plupart du temps, la blague vise l'autre. Les cas de figure qui seront énoncés plus loin en sont la preuve. Or, les mauvaises blagues se comptent par milliers et n'apportent aucun soulagement ni mieux-être aux gens qui en sont les victimes. Ainsi d'une blague sur les trisomiques qui valut au comédien Patrick Timsit - qui n'en était pas l'auteur - d'être fustigé par les parents d'handicapés, avant qu'ils ne lui pardonnent. De cette mésaventure naquit l'association «Notre regard» qui s'efforce de «faire sortir les handicapés mentaux de leur ghetto »; Timsit en est l'un des deux vice-présidents.

Gilbert Collard et Denis Trossero (2000), auteurs de Peut-on rire de tout? se demandent: «Si le rire est le propre de l'homme, est-il toujours propre de rire de l'homme? » Celui qui rit de soi peut plus facilement rire de l'homme. Or, plusieurs humoristes officiels auraient le sens de l'humour "pour l'autre», mais bien de la difficulté à rire lorsque l'on s'attaque à un trait de leur personnalité ou à un aspect de leur vie privée. Collard et Trossero avancent que «l'humour est doute permanent» (p. 28); le doute est probablement la mesure inconsciente qui guide la création, et encore plus la diffusion, d'un bon mot, d'une histoire, d'un récit humoristique, voire d'une blague.

Dans les pages qui suivent, nous verrons comment les humoristes québécois et français abordent la maladie mortelle, 
la vieillesse et la mort par le biais de l'humour. Nous nous attarderons surtout au film Pourquoi moi? / Why me ? $^{3}$ de Janet Perlman et Derek Lamb pour ce qui est des réactions des patients à l'annonce d'une maladie mortelle, et aux monologues d'Yvon Deschamps pour ce qui touche à la vieillesse et à la mort ellemême. Nous confronterons leur approche humoristique aux théories de médecins et psychanalystes spécialistes dans ces domaines.

\section{LE C/A/N/C/E/R}

«Noël au scanner, Pâques au cimetière ", ironisait Pierre Desproges, humoriste français atteint d'un cancer. Cette formulation brève et cinglante exprimait son peu d'espoir d'y survivre.

De toutes les maladies mortelles, le cancer est, encore aujourd'hui, l'une des plus communes, du moins en Occident. Au Canada, où, la plupart du temps, le cabinet du médecin ne rappelle un patient que s'il y a l'obligation de passer de nouveaux tests ou s'il doit lui annoncer une «mauvaise nouvelle», la peur du cancer est tellement répandue, qu'automatiquement, le patient appelé songera qu'il en est probablement atteint. Peut-on l'en blâmer quand on sait que le cancer est la première cause de mort prématurée, et qu'il dispute cette triste place aux maladies du cœur comme première cause de mortalité chez les adultes en général ${ }^{4}$ ?

Certains humoristes mettent en scène des personnages hypocondriaques aux aguets du moindre signe d'une maladie qui leur vaudrait beaucoup d'attention et de petits soins, rendant celle-ci presque enviable. Songeons au personnage de Denise dans le monologue de Clémence Desrochers, "Me semble que j'ai une bosse». À la découverte d'une excroissance sous un sein, Denise s'empresse de téléphoner à sa mère qui, contrairement à elle, n'a pas vu de gynécologue depuis vingt ans. Sa mère ne semblant pas recueillir la confidence de sa fille avec assez de sérieux, Denise décide de prendre rendez-vous avec une femme médecin et, face au délai d'un mois qui l'attend, s'écrie: "Quoi, pas avant un mois? Mais il va m'en pousser d'autres. Ça veut dire que je ne saurai pas avant un mois si j'ai le C/A/N/C/E/R? Mais je vais vivre un vrai $\mathrm{C} / \mathrm{A} / \mathrm{L} / \mathrm{V} / \mathrm{E} / \mathrm{R}$. (Elle raccroche.) J'pars-tu faire le tour de la terre où j'm'en vas-tu rester chez ma mère ${ }^{5}$ ? » (1995, p. 199-201).

Désir inconscient d'attirer l'attention ou frayeur réelle? Il est difficile de le percevoir au début du monologue. Le besoin d'attention est évident, mais à mesure que le monologue se poursuit, croît la peur $\mathrm{du}$ diagnostic. Tout ce que font les pro- ches prend une couleur curieuse: la mère a envoyé trop de fleurs, la sœur a trop baissé l'éclairage partout dans la maison. Denise les soupçonne d'avoir parlé avec le médecin et d'en savoir plus qu'elle au sujet de son état de santé. Elle déclare:

En tout cas, si j'ai le C/A/N/C/E/R, écoute bien ce que j'vas te dire; j'veux pas en entendre parler, j'en parlerai pas à personne.

Parlez-moi z-en pas.

J'en ai déjà parlé à Hélène, à sa mère, à sa sœur, au coiffeur, au pharmacien, à la dépanneuse, à la caissière à banque.

Puis, elle appelle le cabinet du médecin pour obtenir les résultats :

\section{Négatif? (pause ) \\ C'est-à-dire? \\ Le contraire de positif. (pause) \\ C'est à-dire?}

Pourriez-vous m'expliquer autrement

la même chose, parce que j'voudrais

l'expliquer à ma à ma à ma mère.

Vous êtes bien certaine?

Quelle bonne nouvelle!

Pendant que je vous ai docteur,

l'autre, vous l'avez vérifié aussi?

À la fin, elle lance un grand cri

de soulagement.

(1995 p. 200-201)

Le cancer est encore aujourd'hui, pour la majeure partie de la population, la maladie qui effraie le plus, probablement parce que l'individu n'est jamais certain de pouvoir s'en débarrasser tout à fait, malgré tous les traitements qu'il subira. S'il y a rémission, il y a très souvent rechute ou, pis encore, invasion d'une autre partie du corps - sinon de tout le corps - par les métastases. Le cancer est une maladie tellement sournoise, qui emprunte tant de formes, qu'il évoque l'hydre à mille têtes ; une maladie aux contours flous, qu'on tente d'éradiquer par des traitements douloureux, qui diminuent la personne physiquement et dont les conséquences secondaires - perte des cheveux, amaigrissement extrême, parmi d'autres - attaquent non seulement l'intégrité physique mais aussi la dignité du malade. La plus redoutée des maladies par le plus grand nombre d'individus, le C A N C E R est imprononçable d'une traite. Chaque lettre doit donc à la fois être détachée et ponctuée par l'humoriste, comme si le fait de ne pas dire cancer tout simplement pouvait tenir la maladie en joue.

Peut-être est-ce en soupesant l'anxiété de chacun devant cette maladie - et, bien entendu, devant toute autre maladie mortelle - que les cinéastes Janet Perlman et Derek Lamb ont réalisé Pourquoi moi?, un film d'animation de neuf minutes, qui montre les réactions d'un patient à l'annonce par son médecin d'une maladie mortelle innommée qui ne lui laisse qu'une minute à vivre.

Dans son monologue "La maladie», composé entre 1968 et 1969, Deschamps décrit une famille où l'une des grand-mères commente sans cesse les maladies «des autres » tout en interdisant aux membres de la famille de parler de maladie, parce que «inque à en entendre parler, ça l'aurait rendue malade [...] À longeait les murs du passage pis à nous watchait au cas y'où c'qu'y en aurait eu un, p't-être, qui aurait eu l'intention de tomber malade».

Totalement surréaliste, ce monologue nous introduit à la honte et au refus pathologique de la maladie et de la mort. Lorsque la grand-mère se décide à appeler le médecin de famille, c'est, dans tous les cas, trop tard; le «non malade» ne respire plus. En général, cela fait entre quatre et cinq semaines qu'il ne s'est pas levé, mais la grand-mère tient à s'assurer qu'il n'agit pas ainsi par simple paresse! Au médecin qui ne comprend pas qu'on l'appelle toujours trop tard, la grand-mère rétorque: «On vous aurait téléphôné si y avait été malade. Y nous a jeté dans face en pleine santé, c't'homme-là! » Seul le cousin Lucien fait exception. Le médecin de famille, ayant été prévenu par une voisine, passera outre aux objections de la grand-mère et Lucien se retrouvera à l'hôpital : «Ma grand-mère a rasé d'en faire une syncope! Nous faire ça à nous autres! » Le pauvre Lucien vivra encore 17 ans, mais dans la souffrance; la grand-mère y verra un châtiment bien mérité, pour avoir jeté la famille dans la honte.

Leçon retenue: "Y souffra tellement qu'y a jamais un autre qui a osé après lui. Entécas, toués autres ont jamais été malades, pis à mesure qu'les années passaient pis qu'tout l'monde mourait en pleine santé, ma grand-mère rougissait d'orgueil. [...] Après Lucien, toués autres sont morts d'leur belle mort. Quarante et un en neuf ans. C'est pas beau, ça?»

Or, cette grand-mère, "Qui avait passé 107 ans d'sa vie à s'occuper d'la santé des autres, han! À voir à c'que jamais personne soit malade, avant d'mourir, à été malade comme un chien! [...] Ça faisait tellement pitié de nous faire ça, à cassé toute not'fun les trois jours qu'on a été au salon mortuaire»(1998, p. 44-50). En effet, la famille avait tendance à s'amuser ferme dans les salons mortuaires car ses morts étant décédés «de leur belle mort», il n'y avait pas de quoi pleurer. De plus, les membres de la famille adoraient tourmenter les autres clients du salon en leur demandant de quoi leur disparu était mort et se vanter auprès d'eux de leur bonne fortune. 


\section{LA FONCTION CATHARTIQUE DU RIRE}

Contrairement au monologue de Clémence Desrochers, qui se moque gentiment des hypocondriaques, Pourquoi moi? /Why me? n'offre pas d'issue heureuse: la maladie est irrévocable. Les cinéastes Perlman et Lamb semblent plutôt vouloir attribuer au film une valeur cathartique pour les gens atteints de maladie grave et leurs proches, qui ne comprennent pas toujours les réactions du patient. Dans le film, ce dernier passe par toutes les phases «normales» qui suivent une telle annonce et qui, selon Kübler-Ross et Kessler, ressemblent à celles que l'on a devant une tare chez nos enfants ou la perte d'un objet, peu importe la gravité du cas: le refus/la colère/le marchandage/la dépression/l'acceptation (2002, p. 83-84).

Dans le premier cas cité en exemple par les auteurs, il s'agit de la réaction de parents apprenant que leur nouveau-né est aveugle; dans le second, d'une personne qui perd ses lentilles cornéennes. Dans Pourquoi moi? on retrouve exactement ces cinq phases : incrédulité face au diagnostic, expression de la colère contre le «mauvais» médecin, croyance en un nouveau remède ou en un miracle (cryogénie), apitoiement sur son triste sort du fait que sa mort ne touchera personne, enfin, acceptation de la situation doublée de la volonté de tout mettre en ordre avant de partir et même de profiter de chaque seconde qui reste.

Kübler-Ross et Kessler soulignent toutefois que «tout le monde ne passe pas par ces cinq étapes à chaque accident de la vie, et que les réactions ne suivent pas toujours le même ordre, certains stades pouvant même être vécus à plusieurs reprises...». Ils concluent: "Plus on a l'expérience du détachement, mieux on est armé pour affronter la vie» (2002, p. 84). Ou le deuil, pourrait-on ajouter, puisque c'est vraiment de cela qu'on a peur: du deuil de soi, de sa propre vie. Le titre du film est porteur de la peur et de l'injustice dont on se sent victime devant la mort précipitée. L'humour, ici, a une visée cathartique face à l'inéluctable, tout comme l'était, probablement, l'humour grinçant que Desproges retourna contre lui-même lorsqu'il se sut atteint d'un cancer: «Plus cancéreux que moi, tumeur ${ }^{6}$ !»

\section{LE DEUIL DE SOI}

L'œuvre de Woody Allen, qu'elle soit cinématographique ou écrite, est traversée par l'angoisse de la mort. Des dizaines d'aphorismes nous transmettent cette angoisse. "Le lit est le terrain d'entraînement du cercueil» nous en donne la

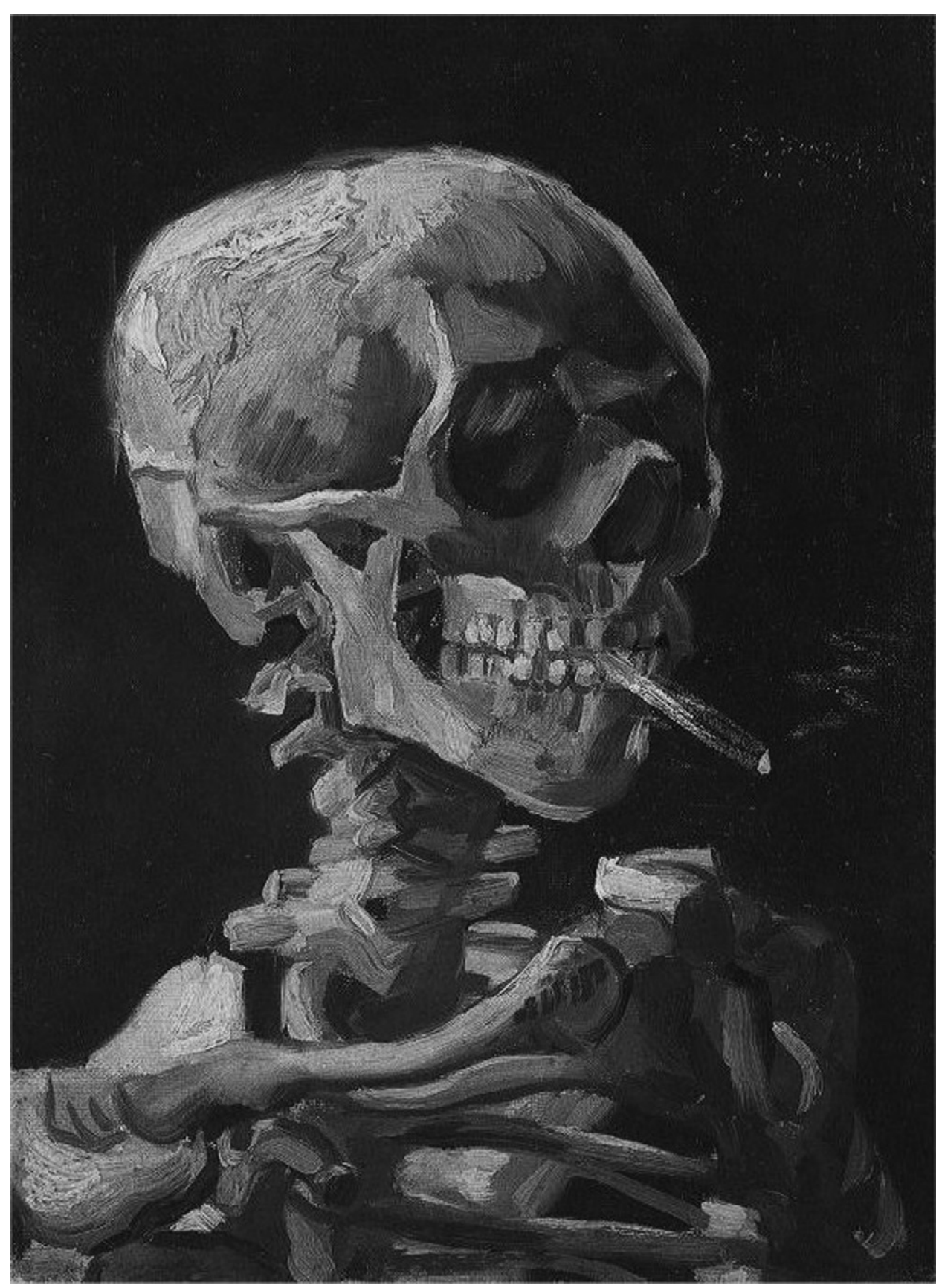

mesure quotidienne; «Je n'ai pas peur de mourir, mais je préfère ne pas être là quand cela va arriver », celle de la distance que toute personne souhaite mettre entre ellemême et la mort ${ }^{7}$.

La peur de la mort conduit l'être humain soit à la nier en n'y pensant jamais, soit à la croire à sa porte à tout instant. Dans le second cas, les gens qui entretiennent un dialogue malsain avec la mort s'examinent, écoutent leur corps et leur cœur et, souvent, consultent les chroniques, les encyclopédies, les sites et les dictionnaires médicaux par peur de tomber malades et surtout de se rendre compte trop tard qu'ils sont atteints d'une maladie mortelle. Mieux vaudrait pour eux de suivre le conseil de l'humoriste français Guy Bedos:

\section{Crâne à la cigarette (1885-86),} Vincent Van Gogh.

Alors, y a un truc qu'il vaut mieux éviter si on veut pas tomber malade, c'est de compulser un dictionnaire médical.

... Parce que là, si vous êtes tant soit peu impressionnable. Si t'es un tout petit peu émotif, tu vois. Ce que tu lis, tu l'as. (1989, p. 60)

Ce que l'être humain a le plus de difficulté à assumer, selon les spécialistes des questions relatives à la mort et au deuil, ce serait « la perte du sentiment d'invulnérabilité propre à la jeunesse», jeunesse qui 
semble reculer à mesure que l'on vieillit. Selon les médecins Kübler-Ross et Kessler: «La plupart d'entre nous refusent le sentiment de perte durant leur vie, car ils ne comprennent pas que celui-ci en fait intrinsèquement partie...» (2002, p. 82). Peut-être, au contraire, le comprennentils trop bien!

Kübler-Ross et Kessler attestent: " Vivre dans l'incertitude d'un possible deuil est en soi un deuil. Quelle que soit l'issue, cela reste un deuil qu'il faut gérer» (p. 89). Or, au plan de l'inconscient, penser sa propre mort relève de l'impossible, selon KüblerRoss. Cette dernière affirme que « la mort a toujours été pour l'homme un objet de répulsion » et qu' « elle le restera toujours». Selon elle, «notre esprit, dans sa partie inconsciente, n'admet pas que nous puissions mourir sans être tués; la mort due à une cause naturelle ou à la vieillesse lui est inconcevable. C'est pourquoi l'idée de mort en soi est associée à celle d'une mauvaise action, d'un événement effrayant, de quelque chose qui, par définition, appelle une vengeance et un châtiment» (1975, p. 10).

S'ajoutent à ces raisons les progrès de la science qui font reculer toujours plus tard l'âge de la mort: "plus la réalité de la mort s'éloigne, plus on la craint et la nie (1975, p. 15) ». Cela semble vrai jusqu'à un certain point, car si l'on craint la mort, il arrive qu'on l'appelle aussi, lorsque, très diminué par l'âge, la vie n'a plus rien d'agréable à nous offrir.

\section{LA MALADIE DE LA VIEILLESSE}

Certains se souviendront sûrement de Jeanne Calment, qui, morte à 122 ans, 5 mois et 14 jours, détient toujours le « record de longévité humaine légalement prouvée» par des actes d'état civil. Ayant gardé bon pied, bon œil au-delà de son $100^{\mathrm{e}}$ anniversaire, elle avait vécu seule jusqu'en sa $111^{e}$ année. En 1996-1997, elle devint célèbre grâce ou à cause de Laurent Gerra qui en fit matière à rire, dans ses chroniques humoristiques à l'émission Studio Gabriel, diffusé ici sur TV5. Gerra la faisait tenter de s'exprimer dans un charabia incompréhensible, se moquant ainsi du disque qu'elle avait enregistré en 1996, à 121 ans, pour financer des minibus pour sa maison de retraite ${ }^{8}$. Il est vrai que les dernières images d'elle, montrées à la télévision lors de son $122^{\mathrm{e}}$ anniversaire, ne donnaient pas à penser qu'elle pût encore avoir l'oreille juste et un reste de voix musical.

Vieillir tourmente les gens. En revanche, la longévité les fascine. Aussi pistet-on de près les doyens et doyennes de l'humanité, qui se succèdent sur le trône de la longévité. Les derniers porteurs du titre de doyens - un Portoricain de 115 ans, puis une Américaine de 114 ans -, sont morts à trois jours d'intervalle. Dans le même communiqué annonçant le décès de l'Américaine, l'agence de presse Reuters était déjà en mesure de donner le nom de la nouvelle doyenne, une Japonaise âgée de 114 ans. C'est qu'il existe un comité international des super-centenaires!

S'il est vrai que les super-centenaires sont des «natures", capables de prendre soin d'eux-mêmes jusqu'à 110 ans environ, la plupart des gens commencent à décliner sérieusement, une fois atteints les 85 ou 90 ans. Ils se retrouvent alors en centre d'accueil. On peut se demander qui jouit ou se réjouit des records de longévité. "Si je deviens centenaire, je me lèverai chaque matin pour lire les faire-part nécrologiques des journaux. Si mon nom n'y est pas, je retournerai me coucher», écrivait Paul Léautaud ${ }^{9}$. Voilà un emploi du temps exaltant!

Yvon Deschamps, un humoriste qui ne soumet sa plume à aucune rectitude, a consacré quelques monologues à la vieillesse. Ils nous permettent de mesurer l'état d'esprit des gens en ce qui concerne les «vieux» et la mort, sur une période de trente ans.

Dans «Pépère », un monologue écrit au début des années soixante-dix, Deschamps rappelle les liens que son alter ego entretenait avec son grand-père, quand il était enfant. Le grand-père vivait alors chez ses parents, le credo de son père étant: «Des fois y est ben achalant mais tant que je s'rai en vie, y s'ra pas dit qu'mon vieux père va moisir à l'hospice. » Le petit Deschamps ne met pas de gants blancs pour dire ses quatre vérités à son grand-père:

J'disais : «Pépère, vous parlez de "dans l'temps" parce que dans c'temps-là, vous étiez capable de faire quelque chose pis que là vous êtes pus bon à rien. C'est toute!» Non c'est vrai, y était ben smatte mais nous autres, y nous servait pus à grand-chose... Y s'berçait un peu, c'est toute. Des fois y allait répondre à porte. Mais fallait l'watcher parce que nous autres, on était habitués, mais le monde qui l'connaissaient pas, des fois y en avaient peur. Eille, y était vieux là, ça pas d'bon sens c't'hommelà! Pis y était paralysé d'un côté pis y shakait d'même pis y était pus beau beau beau à voir... J'sais pas, y se r'tenait pareil. On aurait dit qu'y voulait pas partir. C'est drôle à c't'âge-là les vieux han? Ça s'accroche pis on sait pas pourquoi. (1998, p. 17)
Que sa propre mort soit le deuil le plus difficile à envisager défie toute logique puisque l'homme en est prévenu dès sa naissance. Or, ni le temps, ni la psychanalyse, ni l'avancement des sciences, ni les nouvelles technologies n'ont réussi à vaincre la mort et la peur de la mort. Malgré cette peur, lorsque la santé n'y est plus ou que les personnes âgées ne trouvent plus à s'occuper, elles appellent la mort et se plaignent souvent que Dieu n'entend pas leurs suppliques et ne les appelle pas auprès de lui. Vieillir, oui, mais jusqu'à quand? Ne devrait-il pas y avoir, en ce domaine comme en tout autre, un «juste milieu»?

C'est ce que semblent penser ceux qui entourent les vieux. Dans "Pépère», Deschamps brosse aussi le tableau psychologique des autres membres de la famille: «Pis ma mère à l'aimait ben gros quand $\mathrm{y}$ était d'même: tranquille [...] Des fois à y disait: "Pépère, si l'bon Dieu pouvait v'nir vous chercher, han? Pauvre vieux, vous vous ennuyez de mourir..." " Les enfants lui jouent des tours, parfois mauvais; on l'empêche de sortir pour éviter que les voisins lui crient des noms et qu'après ils rient d'eux.

Les vieux gênent. C'était vrai, hier, mais on le taisait, car on avait de la morale. C'est vrai aujourd'hui, même si, de nos jours, il est rare que les grands-parents demeurent avec leurs enfants et petits-enfants et que la rectitude politique nous dicte toute une série de mots-cache pour adoucir, voire gommer la réalité: les aînés ont remplacé les vieux d'autrefois et on ne les met plus à l'hospice, mais ils choisissent d'entrer en maison ou centre d'accueil. Malgré les mots, la gêne persiste et c'est encore Deschamps qui nous en donne la mesure, dans «Le bénévolat», un monologue pour le moins grinçant:

Ben en faite, y a pus d'personnes âgées là-d'dans, c'est inque des vieux pis des p'tites vieilles. Non, mais les personnes âgées sont pus capables de rentrer, les autres sortent pus. Eille, ça meurt pus! $\mathrm{Y}$ ont sept centenaires, $\mathrm{y}$ en ont 48 en haut de 95! Eille, ça veut pus partir aujourd'hui. Ça vit tellement vieux que ça t'fait pus d'peine quand ça meurt! Même que t'es content, ça fait tellement longtemps que t'attends, t'sais!

Non, soyez honnêtes: tu rentres le vieux à 82 pis y part à 104, 22 ans à aller au centre d'accueil toués fins de semaine, ça casse des fins d'semaine, ça!

(1998, p. 471-472)

Trente ans plus tôt, lorsque le grand-père passait l'arme à gauche, le petit Deschamps s'étonnait de ce qu'il ressentait: 
Après, c'tait pus pareil dans maison. C'est comme si y aurait manqué queque chose, euh... Mon père, lui, y parlait pus, rien. Même moé j'me sentais drôle, t'sais, comme quand tu sens queque chose de bizarre dans l'estomac. C'est dur à expliquer, mais j'me sentais un peu comme quand mon chien s'était faite frapper par un char. (1998, p. 19)

La mort de parents dans un centre d'accueil laisse-t-elle le même goût amer à ceux qui restent? Rien de moins sûr, car encore faudrait-il que les enfants se rappellent de leurs vieux. Deschamps aborde cette défection de la famille par la bouche d'un vieux: "Monsieur, si vous saviez... Moé, chus rendu tellement impotent que j'aimerais pas que mes enfants me voyent comme ça » $(1998$, p. 475).

La manière qu'a Deschamps de décrire la déchéance physique des vieux - santé et beauté - montre aussi les limites de régénération du corps et l'impossibilité pour la science et la cosmétologie d'y remédier au-delà d'un certain seuil: "Quand tu les brasses vite, ça sent drôle! Pis faut qu'tu les déplies tranquillement, ça casse à rien! [...] Moé, avec la peau qui pend, je l'sais plus de quel bord ça plie. Après ça, trouve les lunettes, les cannes, les marchettes... » (1998. p. 472).

Dans ce monologue, Marcel devient agressif envers les gens âgés au point d'envoyer voler la canne d'une petite vieille. La voilà réduite à rentrer au centre d'accueil à quatre pattes sous les «petits coups de pied» de Marcel, qui se fait arrêter et se sent «comme un vrai criminel». Voir trop de gens âgés le déprime. Pour pallier l'agressivité de son mari et l'aider à retrouver du respect et de la compassion pour les personnes âgées, sa femme l'envoie faire du bénévolat au centre d'accueil en question.

Il faut noter dans l'écriture de Deschamps l'utilisation du «ça» pour désigner les nonagénaires: "Ça respire encore, ça?»(1998, p. 474). Il revient à l'infirmière en chef de résumer la «vie» trépidante des pensionnaires, quand elle se veut rassurante pour Marcel: «Y peut rien arriver, toute est faite: $\mathrm{y}$ ont mangé, $\mathrm{y}$ ont été changés pis y sont attachés, y peuvent pas glisser d'leurs chaises [...] Y ont aucune autonomie. Faut les faire manger, y peuvent pas lever le p'tit doigt!»

Paradoxe intéressant que celui du refus et de l'attente de la mort, mouvements qui s'inscrivent tous deux dans la crainte de celle-ci. Nous sommes donc en droit de nous demander si l'annonce d'une maladie mortelle est reçue de la même façon par un jeune patient que par un patient âgé. Toujours selon Kübler-Ross, l'un des

facteurs essentiels qui contribuent au refus de «faire face à la mort calmement [...] tient à ce que l'agonie est, à bien des égards, plus cruelle qu'autrefois et que le mourant, en particulier, est plus seul, car tout est devenu mécanique et sans âme» (1975, p. 16). Tout est fait dans le seul but de l'avancement de la science et non pas pour répondre aux besoins du malade en tant qu'être humain, qu'individu.

La pièce Wit de l'auteure américaine Margaret Edson - écrite en 1991 et refusée par tous les théâtres jusqu'en 1995 -, a été présentée au Théâtre de Quat'Sous, à Montréal, en 2005; elle propose un exemple éloquent de cette mécanisation de la mort. Dans la pièce, l'idée même qu'il doive faire preuve d'un peu d'humanité envers la patiente atteinte d'un cancer ovarien de type 4 avec métastases rebute un jeune chercheur prometteur, pourtant ancien élève de la patiente; chaque fois qu'il s'apprête à quitter la chambre et que son maître lui fait un signe de la tête pour lui rappeler qu'il a oublié quelque chose, le jeune homme murmure: «Shit, l'humain!», ce qui le conduit invariable-

\section{LA PEUR DE LA MORT CONDUIT L'ÊTRE HUMAIN} SOIT À LA NIER EN N'Y PENSANT JAMAIS, SOIT À LA CROIRE À SA PORTE À TOUT INSTANT.

ment à dire des inepties à la patiente et à déclarer à une jeune infirmière que tous les jeunes chercheurs estiment perdre leur temps en suivant le cours obligatoire sur les relations humaines.

Marie-Frédérique Bacqué affirme : «La mort est vécue désormais, non plus comme une fatalité d'ordre biologique, mais comme une violence, une injustice, un aléa, l'échec d'une existence. [...] Ces sentiments ne sont sans doute pas nouveaux, mais jamais comme aujourd'hui, n'ont-ils été autant au cœur de la réflexion, du discours et des usages autour de la mort. On pourrait même caractériser le $\mathrm{XX}^{\mathrm{e}}$ siècle en Occident par sa révolte contre la mort et par l'impuissance de l'homme à en venir à bout, malgré l'augmentation de la longévité et les progrès de la médecine » (2002, p. 9-10). La mort passe ainsi du statut de finitude qu'elle avait autrefois - et qu'elle a toujours dans d'autres cultures - à celui de "maladie incurable», pour reprendre l'expression de Louis-Vincent Thomas (1985, cité dans Bacqué, 2002, p. 33). Les progrès de la science et de la médecine font que l'homme s'imagine, non pas immortel, mais «amortel». Mourir est anormal. Bacqué ajoute :

La mort fait donc l'objet d'une tentative de maîtrise et de déni à travers le souhait d'un "court-circuit» final, exemplarisé par l'infarctus du myocarde ou la rupture d'anévrisme. Ce serait une «mort idéale», permettant de s'éteindre brutalement, ou dans son sommeil, en évitant la souffrance et la déchéance, l'agonie et le questionnement anxiogène; une mort ponctuelle qui mettrait d'emblée la note finale à une vie, proprement, sans atermoiement, comme en plein élan. Or la «bonne mort», au Moyen

Âge, correspondait exactement au contraire: il fallait un temps de préparation, un temps de communication pour les proches, un temps de travail spirituel sur soi et sur ce qu'on allait laisser. (2002, p. 34-35)

Le manque de temps, c'est ce qui fait défaut à l'homme ordinaire du film Pourquoi moi? de Perlman et Lamb. Les quelques minutes qu'il lui reste sont, pour le personnage principal du film, une méta- phore de la hâte dans laquelle on vit, mais elles sont aussi l'expression de la «surprise de la mort». Cinq minutes à vivre, mais pas une question sur la mort. Déni, colère, marchandage... Lorsque arrive «l'acceptation », c'est celle de vivre pleinement les quelques secondes restantes, non pas pour se préparer à la mort, mais pour prolonger la vie, pour enfin vivre pleinement jusqu'au bout.

Cela pourrait encore s'apparenter à la «pulsion de vie, en fin de vie» que Michel de M'Uzan a étudiée et encourage (De l'art à la mort, Paris, Gallimard, 1976, cité dans Bacqué, 2002, p. 38-39). Pas une question non plus sur les souffrances qui accompagnent d'ordinaire la maladie. Vrai, le temps écourté peut donner à penser qu'il n'y aura pas de souffrances car il n'y aura ni thérapie ni acharnement thérapeutique. L'homme ordinaire de Pourquoi moi? ressemble aux personnages des films d'animation pour enfants et adultes qui se déroulent dans des univers violents, mais dans lesquels les personnages se dissolvent plutôt que de mourir de manière réelle. Dans notre film, le face-à-face avec la mort 
est évacué; en lieu et place de la mort qui aurait dû être vécue dans la souffrance, c'est la vie exaltée qui aura le dessus. En somme, Pourquoi moi?, tout en révélant les réactions normales du patient devant un diagnostic morbide, fait surtout passer le message qu'il faut vivre pleinement car nul ne sait quand le couperet de la mort peut s'abattre sur lui.

Pourrait-il en être autrement? Si l'on considère souvent avec un regard critique comment les médecins annoncent la maladie mortelle à leurs patients - qu'ils tournent autour du pot ou qu'ils le fassent de manière abrupte -, on ne remet que très rarement en cause la capacité du patient à recevoir la nouvelle, c'est-à-dire à faire face à l'idée de la mort. Peut-être faut-il croire avec Marie-Frédérique Bacqué:

[...] qu'irreprésentable inconsciemment, la mort est pensable a minima, et peut, au sein du groupe familial, donner lieu à une parole commune sur le travail de la mémoire. Elle ne peut rester l'issue d'un très grand vieillissement qui fait peur ou la conséquence effrayante d'une catastrophe. Parler de la mort, avant qu'elle se produise, revient, [...] à poser un repérage sur cet irreprésentable. (2002, p. 14)

Parmi les auteurs que nous avons consultés, seule Bernadette Lamboy atteste que l'humain demeure sensible à sa finitude. Dans La mort réconciliée, elle énonce: «En même temps que l'être humain s'éveille à la conscience de sa propre existence, lui apparaît sa lointaine mais non moins inéluctable finitude. La mort, toujours, se présente comme une interrogation pressante» (1989, p. 7). L'auteure reconnaît toutefois qu'au $\mathrm{XX}^{\mathrm{e}}$ siècle, la société occidentale a "cherché à évacuer la mort» (1989 p. 9). Comme la mort se montre plus forte que l'homme, Lamboy espère que surviendra un changement en profondeur: "La mort sera toujours la fin d'une vie, mais elle sera bien davantage: elle viendra à s'inscrire comme dynamisme essentiel de la Vie. Est-ce à dire qu'il faille réhabiliter la mort? Certainement. Mais avant tout, il s'agit de réhabiliter la vie, de réhabiliter la Vie»(1989 p. 8).

Et si le rire faisait partie de cette réhabilitation? On prête à Desproges le libellé de la dépêche annonçant sa mort: «Pierre Desproges est mort d'un cancer. Étonnant, non $^{10}$ ? "Selon Alain Woodrow, "[a]vec "l'humour noir" on touche le lien étroit qui existe entre l'humour et la mort: derrière le détachement enjoué de l'humoriste se profile un certain sentiment tragique de la vie, estime Freud»(2000, p. 111). Le rire tragique va donc au-delà de la mort. Impossible d'aller plus loin!

\section{Bibliographie}

BACQUÉ, M.-F. (2002). Appprivoiser la mort, Paris, Odile Jacob.

BEDOS, G. (1989). Petites drôleries et autres méchancetés sans importance, Paris, Éditions du Seuil.

COLLARD, G. et D. TROSSERO (2000). Peut-on rire de tout? Lausanne, Favre.

DESCHAMPS, Y. (1998). Tout Deschamps, Montréal, Lanctôt Éditeur.

DESROCHERS, C. (1995). Tout Clémence, Tome II : 1957-1994, Montréal, VLB Éditeur.

KÜBLER-ROSS, E. (1975). Les derniers instants de la vie, Genève, Éditions Labor et Fides.

KÜBLER-ROSS, E. et D. KESSLER (2002). Leçons de vie, Paris, JC Lattès.

LAMBOY, B. (1989). La mort réconciliée, La Varenne Saint-Hilaire, Les Éditions Seveyrat.

WOODROW, A. (2000). Et ça vous fait rire!, Paris, Éditions du Félin.

\section{Filmographie}

PERLMAN, J. et D. LAMB (1980). Pourquoi moi? Why Me? film d'animation, Office national du film du Canada, 9 min 35 s.

\section{Notes}

1. Woody Allen, repris par Alain Woodrow dans Et ça vous fait rire!, Paris, Éditions du Félin, 2000. Dans une note, l'auteur relève plusieurs variantes, attribuées à des auteurs tels Alphonse Allais ou George Bernard Shaw, p. 184.

2. Par exemple, Jacques et Novembre (Québec, 1984, de Jean Beaudry et François Bouvier); Journal intime (Nanni Moretti, Italie, 1994); Nick's Movie (Wim Wenders, AllemagneÉtats-Unis, 1980); Le temps qui reste (François Ozon, France, 2005) et bien d'autres encore. La mort en direct (Bertrand Tavernier, France-Allemagne-GrandeBretagne, 1980) se situe un peu à la marge de cette tendance puisque ce film vise surtout à dénigrer la téléréalité.

3. Dorénavant ramené à Pourquoi moi ?

4. Selon les statistiques canadiennes de 2006 sur le cancer, rapportées par la Société canadienne du cancer sur son site Internet, « 2,4\% des Canadiens et 2,7\% des Canadiennes ont reçu un diagnostic de cancer au cours des 15 dernières années. »

5. Les extraits tirés des monologues de Clémence Desrochers et d'Yvon Deschamps respectent l'orthographe originale, telle qu'on la trouve dans leurs livres. Il s'agit de textes écrits pour la scène, donc faits pour être dits.

6. Desproges sur le site Internet $<$ http://pascal. bleuyard.free.fr/laed/index.php/2006/06/07/ plus-cancereux-que-moi-tumeur $>$.

7. Allen cité dans Alain Woodrow, Et ça vous fait rire!, Paris, Éditions du Félin, 2000, p. 184.

8. Informations prises sur le site Wikipédia $<$ http://fr.wikipedia.org/wiki/Pierre_ Desproges>.

9. Citation trouvée sur le site Internet $<$ http:// www.dicocitations.com/auteur/2809/ Leautaud_Paul.php?id=2809\&debut=50>.

10. Selon l'article de Wikipédia (<http://fr. wikipedia.org/wiki/Pierre_Desproges $>$ ), elle serait de la main de Jean-Louis Fournier. 